\title{
SOBREVIVÊNCIA DE ALEVINOS DE JUNDIÁ (Rhamdia quelen QUOY \& GAIMARD, 1824) À VARIAÇÃO DE SALINIDADE DA ÁGUA ${ }^{1}$
}

\author{
SURVIVAL OF FINGERLINGS OF THE JUNDIÁ (Rhamdia quelen \\ QUOY \& GAIMARD, 1824) TO CHANGES ON WATER SALINITY
}

\author{
Maria Ignez Marchioro $^{2}$ Bernardo Baldisserotto $^{3}$
}

RESUMO

O objetivo deste trabalho foi testar a sobrevivência de alevinos de jundiá a diferentes concentrações de salinidade (sal marinho comum e água do mar), pois o sal é indicado no tratamento de algumas doenças e também para reduzir o estresse durante o manuseio e transporte de peixes. Os alevinos $(1,68 \pm$ $0,87 \mathrm{~g}$ ) permaneceram de 3 a 7 dias em tanques de $1400 l$, com água corrente e temperatura entre 23 e $25^{\circ} \mathrm{C}$, sendo alimentados diariamente. Após esse período, foram colocados 50 alevinos em tanques de 250l, com circulação fechada, biofiltro e aeração constante. A concentração da água nos tanques foi de 8,0; 9,0; 9,25; 9,50 e 10,0g/l de sal marinho comum ou 10,0;12,0 e 14,0\% de água do mar artificial. A sobrevivência dos alevinos foi observada ao longo de 96h. Para cada grupo de testes houve um grupo controle nas mesmas condições, mas com salinidade de 0\%o. Não houve mortalidade e alteração do comportamento nos grupos expostos a $8 \mathrm{~g} / \mathrm{l}$ sal marinho comum e a $10 \%$ água do mar. O grupo exposto a $9 \mathrm{~g} / \mathrm{l}$ sal marinho comum também não apresentou mortalidade, mas houve alteração do comportamento alimentar. O aumento da concentração de sal marinho comum ou da água do mar causou uma elevação da porcentagem de mortalidade e alteração do comportamento alimentar. Como $\boldsymbol{R}$. quelen suportou sal marinho comum até $9 \mathrm{~g} / \mathrm{l}$ pelo período de $96 \mathrm{~h}$, o mesmo pode ser testado na prevenção ou tratamento de doenças e na redução do estresse durante o transporte.

Palavras-chave: sal comum, teleósteo, piscicultura, água do mar.

\section{SUMMARY}

The aim of this study was to analyze the survival of fingerlings of Rhamdia quelen to different concentrations of salinity (common marine salt and seawater). Salt is used in the

\begin{abstract}
treatment of some fish diseases and to reduce the stress of handling and transport. Fingerlings $(1.68 \pm 0.87 \mathrm{~g})$ were kept in a 1400 lank with running freshwater at $23-25^{\circ} \mathrm{C}$, for 3 to 7 days, being fed daily. Later groups of 50 specimens were transferred to $250 l$ tanks with a water re-use system. The water concentration was 8.0, 9.0, 9.25, 9.5, or 10.0 / / common marine salt or 10.0, 12.0, or $14.0 \%$ artificial seawater. Survival was observed over a period of 96h. The control group was maintained in the same conditions, but with salinity of $0 \%$. There was no mortality and change of behavior in the groups exposed to $8 \mathrm{~g} / \mathrm{l}$ common marine salt and to $10 \%$ seawater. The group exposed to $9 \mathrm{~g} / \mathrm{l}$ common marine salt also did not show mortality, but there was an alteration of the feeding behavior. Higher concentrations of common marine salt or seawater increased mortality and provoked changes in the feeding behavior. Since $\boldsymbol{R}$. quelen tolerated common marine salt up to $9 \mathrm{~g} / \mathrm{l}$ for $96 \mathrm{~h}$, salt can be tested to prevent or treat diseases and to reduce the stress of transport.
\end{abstract}

Key words: common salt, teleost, fish culture, seawater

\section{INTRODUÇÃO}

Peixes teleósteos de água doce mantêm seus fluidos corporais hiperosmóticos em relação ao meio externo. Devido a esta diferença de concentração osmótica, ocorre uma entrada de água por osmose e perda passiva de íons. Este problema é resolvido através da produção de uma urina diluída e da absorção de íons monovalentes através das brânquias. (EVANS, 1993).

WURTS (1995) destaca que a adição de sal comum $(\mathrm{NaCl})$ na água reduz a diferença osmó-

\footnotetext{
${ }^{1}$ Extraído da dissertação apresentada pelo primeiro autor para obtenção do grau de mestre em Zootecnia na Universidade Federal de Santa Maria (UFSM).

${ }^{2}$ Bióloga, Mestre em Zootecnia.

${ }^{3}$ Oceanólogo, Doutor, Professor Adjunto, Departamento de Fisiologia, UFSM, 97105-900, Santa Maria, RS. E.mail: bernardo@ccs.ufsm.br. Autor para correspondência.
} 
tica entre o meio externo e o plasma dos peixes, diminuindo o estresse no transporte de alevinos e adultos de várias espécies. No manejo dos reprodutores de Piaractus mesopotamicus (pacu) e Colossoma macropomum (tambaqui) o sal comum é utilizado na concentração de 3g/l (NASCIMENTO \& COLARES DE MELO, 1995) e, segundo LOPES $\boldsymbol{e} t$ al. (1989), reduz à mortalidade de Oreochromis niloticus após exposição à malation (pesticida organofosforado).

O jundiá, Rhamdia quelen, é de grande importância econômica no Rio Grande do Sul, pois é uma espécie nativa aparentemente bem adaptada a diferentes ambientes, amplamente utilizada nos viveiros de piscicultura, e de boa aceitação no mercado consumidor. O presente trabalho teve como objetivo testar a sobrevivência de alevinos de jundiá a ambientes com variação de salinidade (concentração de sal comum e água do mar). Através dos resultados obtidos neste trabalho, poderão ser analisados outros parâmetros relacionados com crescimento, hábitos reprodutivos e o desenvolvimento da espécie em diferentes condições de salinidade, bem como verificar a eficácia do sal comum no tratamento ou prevenção do estresse e de algumas doenças, que podem ser consideradas comuns nesta espécie, como é o caso dos ictioparasitas.

\section{MATERIAL E MÉTODOS}

Os alevinos de jundiá (Rhamdia quelen) foram adquiridos de produtores da região com peso médio1,68 \pm 0,87g, comprimento total médio 5,96 \pm $0,70 \mathrm{~cm}(\mathrm{n}=200)$ e foram mantidos de 3 a 7 dias em tanques de 1.4001 , com água corrente, temperatura entre 23 e $25^{\circ} \mathrm{C}, \mathrm{pH} 6,8$ e alimentação à base de ração comercial para peixes (rações e concentrados TEMBIÚ, com $30 \%$ de proteína bruta, $8 \%$ matéria fibrosa e $7 \%$ matéria mineral). Após o período de aclimatação, grupos de 50 alevinos foram transferidos para caixas de cimento amianto (interiormente foram pintadas com tinta impermeabilizante de cor preta) com capacidade de 2501 , com circulação fechada, biofiltro e aeração constante (temperatura 18 $-24^{\circ} \mathrm{C}$ ).

Para determinar o efeito da adição de sal marinho comum (Biosal), a água das caixas foi previamente ajustada às concentrações de 8,$0 ; 9,0 ; 9,25$; 9,5 e $10,0 \mathrm{~g} / \mathrm{l}$. Para os testes em diferentes concentrações de água do mar foram utilizadas as salinidades de 10, 12 e 14\%o. Estas concentrações foram obtidas a partir da água do mar artificial $(35,2 \%$ ), elaborada segundo POTTS \& PARRY (1964), tendo a seguinte composição (em mM): 2,34 $\mathrm{NaHCO}_{3}, \quad 28,25$
$\mathrm{MgSO}_{4} \cdot 7 \mathrm{H}_{2} \mathrm{O}, \quad 25,32 \quad \mathrm{MgCl}_{2} \cdot 6 \mathrm{H}_{2} \mathrm{O}, \quad 10,23$ $\mathrm{CaCl}_{2} \cdot 2 \mathrm{H}_{2} \mathrm{O}, 9,96 \mathrm{KCl}$ e $467,8 \mathrm{NaCl}$. O grupo controle foi submetido às mesmas condições dos grupos experimentais, mas sem adição de sal comum ou água do mar.

As concentrações letais (CL50) de sal marinho comum e água do mar para 24, 48, 72 e 96h foram calculadas pelo programa "Slide Write Plus" (Advanced Graphics Software, Inc Carlsbad, CA, 92008-9690, USA, 10ª edição, julho 1993).

\section{RESULTADOS}

No grupo controle, não houve mortalidade durante o período analisado (96h) e os animais se alimentaram normalmente. Também não houve mortes nos grupo expostos às concentrações de $8 \mathrm{e}$ $9 \mathrm{~g} / \mathrm{l}$ de sal marinho comum. A partir destas concentrações de sal marinho comum (concentrações de $9,25 ; 9,5$ e $10,0 \mathrm{~g} / \mathrm{l})$, quanto maior a concentração mais elevada a mortalidade (figura 1). Os alevinos expostos a concentrações de $9 \mathrm{~g} / \mathrm{l}$ (inclusive) ou superiores não se alimentaram durante todo o período experimental.

Os alevinos de jundiá sobreviveram à água do mar, na concentração de $10 \%$, pelo período de $96 \mathrm{~h}$, tendo se alimentado normalmente neste período. O aumento da concentração da água do mar (salinidades de 12 e 14\%o) aumentou a mortalidade (figura 2), e os alevinos destes grupos não se alimentaram durante todo o período experimental. Os valores calculados da CL50 para sal marinho comum e água do mar nos diferentes tempos estão expressos na tabela 1.

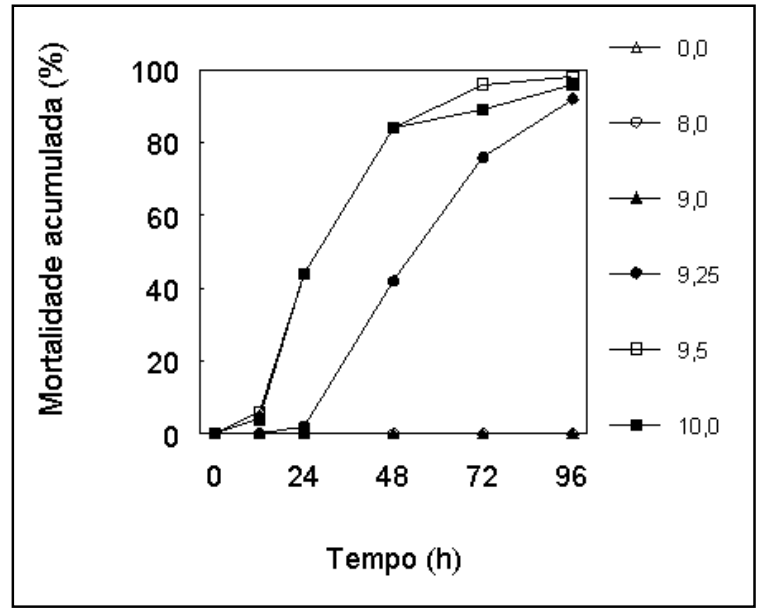

Figura 1 - Mortalidade acumulada em função do tempo em diferentes concentrações de sal marinho comum (g/l) para alevinos de Rhamdia quelen.

* Há sobreposição de pontos nas concentrações de $0 ; 8$ e $9 \mathrm{~g} / \mathrm{l}$ (mortalidade acumulada $0 \%$ ). 


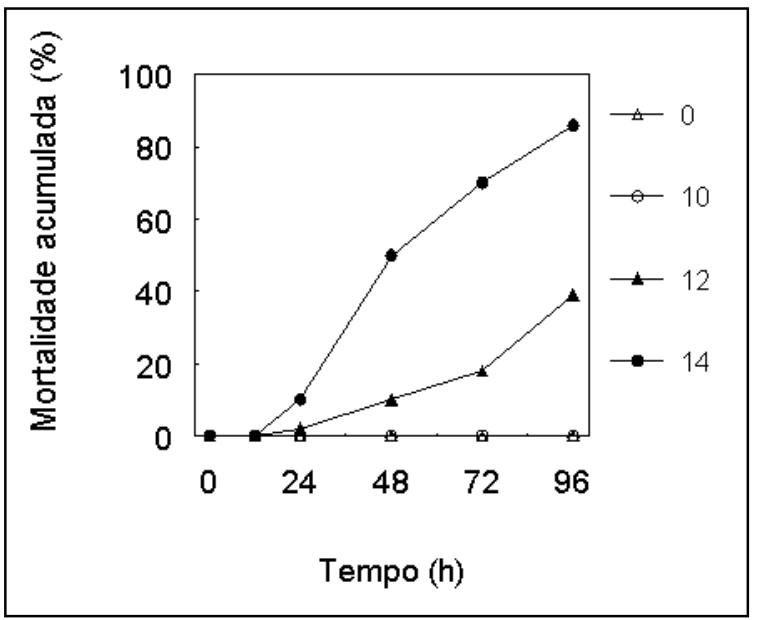

Figura 2 - Percentual de mortalidade em função do tempo em diferentes concentrações de água do mar (\%o) para alevinos de Rhamdia quelen.

* Há sobreposição de pontos nas concentrações 0 e $10 \%$ ( $0 \%)$.

Tabela 1 - Concentração letal (CL50) de sal marinho comum (g/l) e água do mar (\%o) em cada tempo para alevinos de Rhamdia quelen.

\begin{tabular}{ccc}
\hline & $\begin{array}{c}\text { CL50 (sal marinho } \\
\text { comum) }\end{array}$ & CL50 (água do mar) \\
\hline & & \\
48 & 9,94 & 17,66 \\
72 & 9,39 & 13,98 \\
96 & 9,22 & 13,24 \\
& 9,09 & 12,49 \\
\hline
\end{tabular}

\section{DISCUSSÃO}

Nos experimentos com alevinos de $\boldsymbol{R}$. quelen, foi verificado que não há mortalidade com sal marinho comum até $9 \mathrm{~g} / \mathrm{l}$ e concentração da água do mar até $10 \%$ o. Contudo, comparando-se as CL50 de sal marinho comum e da água do mar (tabela 1), pode-se verificar que os alevinos desta espécie toleram uma maior concentração de água do mar do que de sal comum. Esta maior tolerância à água do mar, provavelmente, está relacionada com o fato que o sal marinho comum, utilizado nestes experimentos, apresenta uma maior proporção de $\mathrm{NaCl}$ em relação aos outros íons (o sal é obtido da evaporação da água do mar, mas é misturado com o sal oriundo de minas de sal, com grande proporção de $\mathrm{NaCl}$ ). Esta elevada concentração de $\mathrm{NaCl}$ dificulta a manutenção do equilíbrio osmótico dos alevinos. Exemplares de Oreochromis mossambicus (ex-Tilapia mossambica) também apresentam um padrão semelhante: toleram água do mar a 35\% (BALDISSEROTTO $\boldsymbol{e} t$ al., 1994), mas só resistem a $17 \mathrm{~g} / \mathrm{l}$ de sal comum $(\mathrm{NaCl})$ (TAKASUGI \& BERN, 1962).

Os valores de tolerância à água do mar observados para os alevinos de $\boldsymbol{R}$. quelen estão próximos aos encontrados para outras espécies de "catfish", como Ictalurus cyprinella e Ictalurus niger (PETERSON \& MEADOR, 1994). Segundo os mesmos autores, os ovos de 3 dias de Ictalurus punctatus toleram salinidades de $16 \%$, e, após a eclosão, a tolerância à salinidade cai para $8 \%$. O mesmo ocorre com I. cyprinella e I. niger: os ovos toleram condições mais salinas (15\%o) que os juvenis (8\%o), mas em estágios juvenis mais adiantados, a tolerância aumenta (12\%o), e quando o peixe se aproxima de um ano de idade decresce novamente (10\%o). Valores semelhantes foram encontrados para Heteropneustes fossilis que sobrevive até salinidades de $12,5 \%$ o sem mortalidade até um período de 14 dias (PARWEZ et al., 1979).

Exemplares de Acipenser brevirostrum, testados em diferentes salinidades por diferentes períodos, demonstraram que exemplares com 17 dias de idade toleram salinidades até $5 \%$ o, por $96 \mathrm{~h}$, sem mortalidade. Contudo, peixes com 39 dias exibiram sobrevivência de $100 \%$ em $7 \%$ e $80 \%$ em 9\%o (96h). Exemplares com 63 dias, em salinidade de 15\%o, apresentaram sobrevivência de 53\% após 48 h, e, em $20 \%$, mostram sinais de estresse quase imediato no início do tratamento, com mortalidade de $100 \% \mathrm{em}$ 6 h. Peixes com 76 dias testados em 7 e 9\%o apresentaram sobrevivência de $67 \%$ e, em 11 e $13 \%$, a sobrevivência foi de 5\% até o final do período (96h). Para peixes estocados em salinidades maiores que $15 \%$ o mortalidade foi total no período de $96 \mathrm{~h}$, em todas as idades testadas (JENKINS et al., 1993). Com esses dados, observa-se que a sobrevivência pode variar de acordo com a idade da espécie, por isso, futuramente, será de grande importância testar a sobrevivência do jundiá em função da adição de sal na água em diferentes fases da vida (larva, adulto), bem como a realização de trabalhos sobre o crescimento.

Os testes de sobrevivência com sal comum são úteis, porque considera-se que adição do mesmo na água pode reduzir o estresse durante o transporte de alevinos ou de adultos de um local para outro. A quantidade de cloreto de sódio utilizada no preparo das soluções é muito discutida, podendo variar de 5 a 8g/l (WURTS, 1995). Para PRIETO (1991), o sal comum pode ser utilizado preventivamente como desinfetante de incubadoras e caixas para transporte de peixes, e também apresenta ação curativa contra ictioparasitas, devendo ser utilizado na quantidade de $1-3 \%$. Como $R$. quelen suporta 
concentrações de até $9 \mathrm{~g} / \mathrm{l}$ pelo período de $96 \mathrm{~h}$, o sal comum $(\mathrm{NaCl})$ pode ser testado na prevenção ou tratamento de doenças e na redução do estresse durante o transporte. Contudo, deve-se destacar que é mais seguro utilizar concentrações abaixo de $9 \mathrm{~g} / \mathrm{l}$ nos tratamentos, pois a CL50 para sal marinho comum em $96 \mathrm{~h}$ é muito próxima deste valor $(9,09 \mathrm{~g} / \mathrm{l})$, e os alevinos não se alimentam quando expostos a esta concentração.

\section{REFERÊNCIAS BIBLIOGRÁFICAS}

BALDISSEROTTO, B., MIMURA, O.M, SALOMÃO, L.C Urophyseal control of plasma ionic concentration in Oreochromis mossambicus (PISCES) exposed to osmotic stress. Ciência e Natura, Santa Maria, v. 16, p. 39-50, 1994.

EVANS, D.H. The physiology of fishes. Florida: CRC Press, 1993, 493p. Cap. 11, p. 315-342.

JENKINS, W.E., SMITH, T.I.J., HEYWARD, L.D. et al. Tolerance of shortnose sturgeon, Acipenser brevirostrum, juveniles to different salinity and dissolved oxygen concentrations. Proceedings of the Annual Conference of Southeast Association of Fisheries and Wildlife Agencies, v. 47, p. $476-484,1993$.

LOPES, L.R., SANTOS, S., DELL'AQUA et al. Recovery facilitation of malathion-intoxicated freshwater fish by esposure to hypertonic environment. Brazilian Journal of Medical and Biological Research, v. 22, p. 269-273, 1989.

NASCIMENTO, V.M. da C., COLARES DE MELO, J.S Sobrevivência de pacu, tambaqui e seus híbridos - paqui e tambacu - no período de inverno. In: Relatório Anual do Projeto IDRC/IBAMA. Pirassununga: CEPTA, p. 52-55, 1995.

PARWEZ, I., GOSWAMI, S.V., SUNDARARAJ, B.I. Salinity tolerance of fresh water catfish Heteropneustes fossilis (Bloch). Indian Journal of Experimental Biology, v. 17, p. 810-811, 1979.

PETERSON, M.S., MEADOR, M.R. Effect of salinity on freshwater fishes in coastal plain, drainage in the southeastern U.S. Reviews in Fisheries Science, v. 2, n. 2 p. 95-121, 1994.

POTTS, W.T.W., PARRY, G. Osmotic and ionic regulation in animals. Oxford: Pergamon Press, 1964, 423 p.

PRIETO, A. Manual para la prevención y del tratamiento de enfermedades en peces de cultivo en agua dulce. Santiago: ONU/FAO, 1991, 65 p.

TAKASUGI, N., BERN, H.A. Experimental studies on the caudal neurosecretory system of Tilapia mossambica. Comparative Biochemistry and Physiology, v. 6, p. 289-303, 1962.

WURTS, W. A. Using salt to reduce handling stress in channel catfish. World Aquaculture, v. 26, n. 3, p. 80-81, 1995.

Ciência Rural, v. 29, n. 2, 1999. 